\title{
Clinical Study \\ Sleep Quality and Levodopa Intestinal Gel Infusion in Parkinson's Disease: A Pilot Study
}

\author{
Oriol De Fabregues $\mathbb{D D}^{1}{ }^{1}$ Alex Ferré, ${ }^{2}$ Odile Romero, ${ }^{2}$ Manuel Quintana, ${ }^{1}$ \\ and José Álvarez-Sabin ${ }^{1}$ \\ ${ }^{1}$ Movement Disorders Unit, Department of Neurology, Hospital Universitari Vall D'Hebron, \\ Neurodegenerative Diseases Research Group-Vall D'Hebron Research Institute, Universitat Autònoma de Barcelona, \\ Barcelona, Spain \\ ${ }^{2}$ Sleep Unit, Department of Neurophysiology, Hospital Universitari Vall D’Hebron, Barcelona, Spain
}

Correspondence should be addressed to Oriol De Fabregues; odefabregues@gmail.com

Received 15 June 2018; Revised 8 September 2018; Accepted 14 October 2018; Published 1 November 2018

Academic Editor: Cristine Alves da Costa

Copyright (c) 2018 Oriol De Fabregues et al. This is an open access article distributed under the Creative Commons Attribution License, which permits unrestricted use, distribution, and reproduction in any medium, provided the original work is properly cited.

\begin{abstract}
Background. Sleep problems in patients with advanced Parkinson's disease (PD) have a deleterious impact on quality of life. Objective. To assess the effect of levodopa-carbidopa intestinal gel (LCIG) infusion on sleep quality in advanced PD patients. Methods. Seven patients participated in a prospective pilot study. Before and after 6 months of LCIG infusion, an overnight polysomnography was performed and the Epworth Sleepiness Scale, fatigue scale, Pittsburgh Sleep Quality Index, Beck Depression Inventory, and the Hamilton Anxiety Rating Scale were administered. Results. PSG showed low sleep efficiency. REM sleep without atony was found in 5 patients. After 6 months of LCIG infusion, the percentage of REM sleep decreased as well as the number of arousals especially due to reduction of spontaneous arousals and periodic leg movements during REM sleep, but differences were not statistically significant. Also, scores of all study questionnaires showed a tendency to improve. Conclusion. The results show a trend toward an improvement of sleep quality after 6 months of LCIG infusion, although differences as compared to pretreatment values were not statistically significant. The sleep architecture was not modified by LCIG. Further studies with larger study samples are needed to confirm these preliminary findings.
\end{abstract}

\section{Introduction}

Sleep problems are important nonmotor manifestations in patients with Parkinson's disease (PD) at different stages during the course of the disease. Numerous forms of alterations of physiologic sleep patterns have been reported, ranging from increased daytime sleepiness after introduction of a dopamine agonist to the therapeutic regimen to specific sleep-related diagnoses (e.g., restless legs syndrome, rapid eye movement sleep behavior disorder, and periodic limb movements in sleep) or sleep-related breathing disorders (e.g., obstructive sleep apnea) [1-3]. The origin of these sleep disorders is multifactorial including degeneration of the brain areas that modulate sleep, the symptoms of the disease, and the effect of medications [2].
These disturbances can primarily affect the patient's quality of life and may worsen the symptoms of PD $[4,5]$. In studies that examine the impact of PD on quality of life, sleep difficulties are independent an important predictor of poor quality of life [6]. In fact, sleep disturbance, depression, and lack of independence are the primary determinants of poor quality of life [5]. Also, sleep disturbances contribute to excessive daytime sleepiness and poor daytime functioning as well as patients' reduced enthusiasm for daily events. Adverse effects have also been observed in the sleep habits and the quality of life of their caregivers [7].

There is little evidence of the impact of treatment modalities for advanced PD on sleep [8]. In relation to deep brain stimulation (DBS), a positive effect of subthalamic DBS on sleep-wake disturbances was found in a systematic 
review of 38 studies involving 1443 subjects [9]. However, only seven studies used polysomnography (PSG) to objectively assess sleep parameters. In a small pilot study, pallidal DBS showed improving trends in several PSG measures including sleep efficiency and latency to sleep onset [10]. Continuous levodopa-carbidopa intestinal gel (LCIG) infusion is a therapeutic option for advanced PD patients complicated with motor fluctuations refractory to conventional treatment. It has been shown that LCIG infusion improves nonmotor symptoms and quality of life [11]. The specific effects of LCIG infusion on sleep disturbances have been poorly studied. In a small clinical series of $12 \mathrm{PD}$ patients, subjective measures of sleep quality and daytime sleepiness improved with LCIG infusion, although PSG was not performed [12]. There is only one open-label pilot study with a sample size limited to 11 patients that examined PSG characteristics in PD patients on a stable LCIG dose [13]. Main findings included improvement of subjective sleep quality, motor complications, and activities of daily living. PSG showed a reduction of the number of awakenings in sleep, a trend towards a lower apnea-hypopnea index, and no change in sleep latency, total sleep time and sleep efficiency [13].

The present clinical study was conducted to add evidence of sleep disturbances in advanced PD patients treated with LCIG infusion. The objective was to determine whether treatment with LCIG infusion had a beneficial effect on the quality of sleep in patients with advanced PD. In these patients, an overnight PSG was performed before and after 6 months of LCIG infusion therapy. The quality of sleep is a complex phenomenon, the assessment of which should integrate subjective and quantitative objective measures. For this reason, besides overnight PSG, we also evaluated subjective parameters using a series of validated questionnaires.

\section{Patients and Methods}

2.1. Study Population. Seven consecutive patients with advanced PD were included in a single-center, open-label prospectively pilot study, and were evaluated at baseline and after 6 months of LCIG infusion. The study was conducted in compliance with the ethical standards and was approved by the Ethics Committee for Clinical Research of Hospital Universitari Vall d'Hebron, Barcelona (Spain) and followed the Spanish Law 15/1999 on Personal Character Data Protection concerning confidentiality of Patient's data. The Institutional Review Board Clinical Study registration number was PR(AG)129/2008, and the Clinical Study registration number is NCT03602924. Written informed consent was obtained from all patients.

Patients started LCIG infusion after receiving an implant of percutaneous endoscopic gastrostomy with jejunal extension (PEG-J) following a previously described procedure used in our center [14]. The initial LCIG maintenance dose was calculated according to the levodopa equivalent daily dose; the optimal dose was titrated individually until reaching the best motor performance, controlling motor fluctuations without causing annoying dyskinesia, and getting a stable infusion for less than 16 hours a day, stopped at night, when patients received an oral nocturnal dose of levodopa.

2.2. Study Procedures. All participants underwent a full overnight PSG at the Sleep Unit of our institution. The recorded parameters included electroencephalography (EEG), electro-occulography (EOG), electromyography (EMG), electrocardiography (ECG), respiratory effort, oronasal airflow, oxygen saturation, snoring sounds, and body position. Sleep scoring was performed by a trained technician according to the American Academy of Sleep Medicine (AASM) scoring criteria 2012 [15]. The reported parameters included sleep efficiency, wake after sleep onset (WASO), sleep latency, REM latency, rapid-eye movement (REM) sleep, nonREM (NREM) sleep (stages 1-3), snoring sounds, apnea-hypopnea index (AHI), arousal index, spontaneous arousals, respiratory effort-related arousals (RERA), leg movement arousals, periodic leg movements in sleep (PLMS), PLMS in REM and NREM, oxygen saturation $\left(\mathrm{SpO}_{2}\right)$, and CT90.

2.3. Assessments. Evaluation included the Epworth Sleepiness Scale (ESS) [16], the Fatigue Severity Scale (FSS) [17], the Pittsburgh Sleep Quality Index (PSQI) [18], sleep efficiency, the Beck Depression Inventory (BDI) [19], and the Hamilton Anxiety Rating Scale (HARS) [20]. Also, complete pharmacological data, including antiparkinsonian drugs and treatments potentially influencing sleep and daytime sleepiness (i.e., clonazepam, quetiapine, and serotonin selective reuptake inhibitors) were collected at baseline and at follow-up.

The ESS measures the general level of daytime sleepiness (the sum of 8 item scores, $0-3$ ) with a total score ranging from 0 to 24. The higher the ESS score, the higher the person's average sleep propensity in daily life. The FSS is a 9item questionnaire with questions related to how fatigue interferes with certain activities and rates its severity. Items are scored on a 7 point scale, with $1=$ strongly disagree and 7 $=$ strongly agree, with a total score ranging between 9 and 63 . The higher the score, the greater fatigue severity. The PSQI consists of 19 individual items creating 7 component scores and one composite score. Each item is weighted on a 0-3 interval scale. The global PSQI score is then calculated by totaling the 7 component scores, providing an overall score ranging from 0 to 21 , where lower scores denote a healthier sleep quality. Subjective sleep efficiency (component \#4 of the PSQI) was calculated as number of hours slept/number of hours spent in bed $\times 100$ and expressed as a percentage. The BDI consists of 21 questions about how the subject has been feeling during the last week, and a value of 0 to 3 is assigned for each answer, with total score ranging from 0 to 63 (0-13 minimal, 14-19 mild, 20-28 moderate, 29-63 severe). The HARS consists of 14 items, each of which contains a number of symptoms, which are rated scale of 0 to 4 , with 4 being the most severe. The total score ranges from 0 to 56 .

Moreover, motor fluctuations, dyskinesia, and other motor and nonmotor clinical aspects were evaluated before starting LCIG infusion (baseline) after 6 months of 
treatment. Motor fluctuations were assessed by "off" time, in hours, recorded in Parkinson's Disease Diaryœ. Dyskinesia and other motor symptoms were evaluated using the Unified Parkinson's Disease Rating Scale (UPDRS) [21] part IVComplications of Therapy, UPDRS part II-Activities of Daily Living in On and Off, UPDRS part III-Motor Examination in On and Off; Hoehn and Yahr stage in On and Off [22]; and the Schwab and England Activities of Daily Living (ADL) scale in On [23]. Nonmotor clinical aspects evaluated were cognitive function using the Mini Mental State Examination (MMSE) test [24] and UPDRS part IMental, Behavior, and Mood.

Complete pharmacological data, including antiparkinsonian drugs and treatments potentially influencing sleep and daytime sleepiness (i.e., benzodiazepines (clonazepam, lorazepam), neuroleptic (quetiapine), and serotonin selective reuptake inhibitors) were, recorded at baseline and follow-up.

2.4. Statistical Analysis. Data are expressed as mean and standard deviation $( \pm \mathrm{SD})$. The Wilcoxon signed-rank test was used for the comparison of paired samples before and after 6 months of LCIG infusion. Statistical analysis was performed with the SPSS version 17.0 (Statistical Package for Social Sciences, SPSS, Inc., Chicago, IL, USA). Statistical significance was set at $P<0.05$.

\section{Results}

We studied 6 women and 1 man diagnosed with advanced $\mathrm{PD}$, with a mean age of 69.6 years (range 60-78) and a mean body mass index (BMI) of $24.5 \mathrm{~kg} / \mathrm{m}^{2}$ (range $20-32$ ).

Results of clinical variables are shown in Table 1. A significant improvement in motor fluctuations was observed (daily mean "off" time decreased from $6.3 \pm 1.4 \mathrm{~h}$ at baseline to $1.1 \pm 0.7 \mathrm{~h}$ after 6 months, $P<0.001)$. Motor symptoms evaluated with UPDRS part III remained stable and the mean score in "off" stage did not change in any of the patients. Dyskinesia assessed with UPDRS part IV decreased significantly from $5.4 \pm 2.4$ at baseline to $2.9 \pm 1.1$ after 6 months of LCIG treatment $(P=0.028)$. None of the patients presented a worsening in the percentage of the waking time with dyskinesia, which was reduced in 2 patients $(28.6 \%)$ and remained stable in the remaining 5 patients $(71.4 \%)$. The severity of dyskinesias improved in $3(42.9 \%)$ patients, remained stable in 3 (42.9\%), and worsened in 1 (14.3\%). Nonmotor cognitive function (UPDRS part I) tended to improve.

As shown in Table 2, at 6 months after LCIG infusion, there was a decrease in scores of the ESS, FSS, and PSQI as compared with baseline, indicating an improvement in daytime sleepiness, fatigue, and sleep quality. Severe score of the PSQI at baseline was recorded in 4 patients (57.1\%), 3 of which changed to a moderate score at follow-up. One patient with moderate score at baseline had a normal score at follow-up, and of 2 patients with normal score at baseline, 1 had a mild score at follow-up. Sleep efficiency also improved. Differences, however, were not statistically significant. Changes in the scores of BDI and HARS were not significant either.
TABle 1: Changes in "off” time hours, UPDRS values, cognitive function, body mass index, and pharmacological therapy at baseline and after 6 months of LCIG infusion.

\begin{tabular}{|c|c|c|c|}
\hline Variables & Baseline & $\begin{array}{l}\text { Follow- } \\
\text { up }\end{array}$ & $\begin{array}{c}P \\
\text { value }\end{array}$ \\
\hline $\begin{array}{l}\text { Off time hours recorded in } \\
\text { Parkinson's disease diary® (daily } \\
\text { mean "off" time) }\end{array}$ & $\begin{array}{c}6.3 \pm \\
1.4\end{array}$ & $\begin{array}{c}1.1 \pm \\
0.7\end{array}$ & $<0.001$ \\
\hline UPDRS part IV (dyskinesia) & $\begin{array}{c}5.4 \pm \\
2.4\end{array}$ & $\begin{array}{c}2.9 \pm \\
1.1\end{array}$ & 0.028 \\
\hline \multicolumn{4}{|c|}{ UPDRS part II (activities of daily living) } \\
\hline On & $\begin{array}{l}11.4 \pm \\
5.9\end{array}$ & $\begin{array}{c}10.9 \pm \\
5.6\end{array}$ & 0.742 \\
\hline Off & $\begin{array}{c}22.9 \pm \\
8.3\end{array}$ & $\begin{array}{c}22.3 \pm \\
7.5\end{array}$ & 0.231 \\
\hline \multicolumn{4}{|l|}{ UPDRS part III (motor examination) } \\
\hline 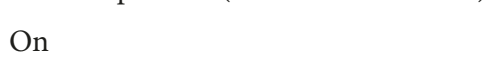 & $18 \pm 3.7$ & $\begin{array}{c}16.6 \pm \\
4.9\end{array}$ & 0.245 \\
\hline Off & $\begin{array}{c}34.1 \pm \\
11.1\end{array}$ & $\begin{array}{c}34.1 \pm \\
11.1\end{array}$ & - \\
\hline $\begin{array}{l}\text { UPDRS part I (mentation, behavior, } \\
\text { and mood) }\end{array}$ & $\begin{array}{c}3.6 \pm \\
3.7\end{array}$ & $\begin{array}{c}2.4 \pm \\
2.1\end{array}$ & 0.156 \\
\hline MMSE (cognitive function) & $\begin{array}{c}29 \\
(27-30)\end{array}$ & $\begin{array}{c}29 \\
(27-30)\end{array}$ & 0.317 \\
\hline Body mass index (BMI) & $\begin{array}{c}24.5 \pm \\
4.0\end{array}$ & $\begin{array}{c}23.8 \pm \\
3.3\end{array}$ & 0.424 \\
\hline \multicolumn{4}{|l|}{ Bedtime drugs, no. patients } \\
\hline Benzodiazepine drugs & $6 / 7$ & $6 / 7$ & 1.000 \\
\hline $\begin{array}{l}\text { Serotonin selective reuptake } \\
\text { inhibitor }\end{array}$ & $4 / 7$ & $4 / 7$ & 1.000 \\
\hline Neuroleptic drugs & $1 / 7$ & $2 / 7$ & 1.000 \\
\hline
\end{tabular}

Data as mean \pm standard deviation unless otherwise stated.

TABLE 2: Changes of subjective parameters before and after 6 months of LCIG infusion therapy in 7 patients with advanced PD.

\begin{tabular}{|c|c|c|c|}
\hline \multirow{2}{*}{ Questionnaires } & \multicolumn{2}{|c|}{ LCIG infusion therapy } & \multirow{2}{*}{$P$ value } \\
\hline & Before & At 6 months & \\
\hline ESS & $6.4 \pm 3.6$ & $4.7 \pm 4.1$ & 0.340 \\
\hline PSQI & $11.9 \pm 6.4$ & 8.9 & .137 \\
\hline Subjective sleep efficiency & $61.9 \pm 21.5$ & $57.8 \pm 16.0$ & 0.612 \\
\hline BDI & $11.7 \pm 8.7$ & $14.3 \pm 9.4$ & 0.497 \\
\hline HARS & $21.6 \pm 10.8$ & $22.1 \pm 12.4$ & 0.917 \\
\hline
\end{tabular}

Data as mean \pm standard deviation. ESS: Epworth Sleepiness Scale; PSQI: Pittsburgh Sleep Quality Index; BDI: Beck Depression Inventory; HARS: Hamilton Anxiety Rating Scale.

Results of PSG showed low generalized sleep efficiency. No significant differences were observed in sleep macrostructure parameters, respiratory events, or periodic leg movements. There was a decrease in the percentage of REM sleep $(16.2 \pm 9.9 \%$ vs. $10.4 \pm 6.8 \%, P=0.080)$ and arousal index (15.0 \pm 7.0 vs. $12.9 \pm 5.6, P=0.115)$ especially due to reduction of spontaneous arousals $(7.5 \pm 3.1$ vs. $5.2 \pm 5.0$, $P=0.075)$ and PLMS during REM sleep (20.7 \pm 31.6 vs. $2.9 \pm$ 3.7, $P=0.285$ ), but differences were not statistically significant. The percentage of NREM sleep increased from a mean of $83.6 \pm 10.1 \%$ at baseline to $89.6 \pm 6.8 \%$ after 6 months of LCIG infusion therapy, although differences did not reach statistical significance $(P=0.080)$ (Table 3$)$. 
TABLE 3: Comparison of polysomnographic results before and after 6 months of LCIG infusion therapy in 7 patients with advanced PD.

\begin{tabular}{|c|c|c|c|}
\hline \multirow{2}{*}{ Variables } & \multicolumn{2}{|c|}{ LCIG infusion therapy } & \multirow{2}{*}{$P$ value } \\
\hline & Before & At 6 months & \\
\hline Sleep efficiency $(\%)$ & $66.7 \pm 8.4$ & $57.8 \pm 16.0$ & 0.173 \\
\hline Wake after sleep onset (WASO) (min) & $119.9 \pm 74.2$ & $113.0 \pm 54.1$ & 0.345 \\
\hline Sleep latency $(\mathrm{min})$ & $29.7 \pm 40.5$ & $71.2 \pm 97.2$ & 0.345 \\
\hline REM latency (min) & $147.3 \pm 72.4$ & $139.8 \pm 63.1$ & 0.893 \\
\hline REM sleep (\%) & $16.2 \pm 9.9$ & $10.4 \pm 6.8$ & 0.080 \\
\hline NREM sleep (\%) & $83.6 \pm 10.2$ & $89.6 \pm 6.8$ & 0.080 \\
\hline Stage 1 & $16.8 \pm 10.0$ & $23.8 \pm 13.7$ & 0.463 \\
\hline Stage 2 & $52.9 \pm 8.4$ & $52.4 \pm 12.0$ & 0.345 \\
\hline Stage 3 & $13.9 \pm 7.5$ & $13.0 \pm 11.9$ & 0.752 \\
\hline Snoring sounds (number/hour) & $323.2 \pm 279.3$ & $228.6 \pm 226.0$ & 0.715 \\
\hline Apnea-hypopnea index (AHI) & $11.8 \pm 18.0$ & $12.7 \pm 14.0$ & 0.686 \\
\hline Arousal index & $15.0 \pm 7.0$ & $12.9 \pm 5.6$ & 0.115 \\
\hline Spontaneous arousals & $7.5 \pm 3.1$ & $5.2 \pm 5.0$ & 0.075 \\
\hline Respiratory effort-related arousals (RERA) & $4.9 \pm 7.2$ & $4.8 \pm 4.1$ & 0.893 \\
\hline Leg movement arousals & $3.1 \pm 2.3$ & $2.9 \pm 2.5$ & 0.462 \\
\hline Periodic leg movement in sleep (PLMS) & $12.5 \pm 11.6$ & $7.7 \pm 11.1$ & 0.345 \\
\hline PLMS in REM sleep & $20.7 \pm 31.6$ & $2.9 \pm 3.7$ & 0.285 \\
\hline PLMS in NREM sleep & $10.3 \pm 9.5$ & $8.3 \pm 12.4$ & 0.893 \\
\hline \multicolumn{4}{|l|}{ Oxygen saturation $\left(\mathrm{SpO}_{2}\right)(\%)$} \\
\hline Baseline & $95.1 \pm 2.3$ & $95.8 \pm 2.1$ & 0.339 \\
\hline Mean & $93.8 \pm 2.0$ & $94.3 \pm 2.2$ & 0.461 \\
\hline Minimum & $87.0 \pm 8.9$ & $86.4 \pm 6.6$ & 0.672 \\
\hline Oxygen saturation <90\%, CT90 (\%) & $2.7 \pm 4.2$ & $1.7 \pm 2.0$ & 0.416 \\
\hline
\end{tabular}

Data as mean \pm standard deviation. 6-10: higher normal daytime sleepiness. 11-12: mild excessive daytime sleepiness. 13-15: moderate excessive daytime sleepiness. 16-24: severe excessive daytime sleepiness.

The patients had no previous treatment at the beginning of the infusion with dopamine agonists. Also, monoamine oxidase (Mao) and catechol-O-methyl transferase (COMT) inhibitors were withdrawn in all patients, whereas the use of other drugs potentially influencing the quality of sleep and daytime sleepiness were allowed. The number of patients taken drugs with hypnotic effect before and after treatment with LCIG infusion remained unchanged (Table 1).

\section{Discussion}

This prospective pilot study in a reduced number of patients with advanced PD treated with LCIG infusion shows that their quality of sleep is poor. Treatment with LCIG infusion did not aggravate the quality of sleep in these patients. We found a decreased time in REM sleep and a tendency of improvement in the number of arousals especially in relation to a reduction of spontaneous arousals and PLMS during REM sleep. Despite a reduction in the percentage of REM sleep, the quality of sleep seems to be better as shown by a decrease of spontaneous arousals and PLMS. These changes in objective parameters may explain the trend towards improvement of subjective measures. Although statistically significant differences in clinical or polysomnographic parameters were not found due to the limited population of 7 patients with advanced $\mathrm{PD}$, the present results regarding maintenance of sleep architecture and a trend toward an improvement of sleep quality after 6 months of LCIG infusion, are similar to results reported by Zibetti et al. [13]. In this respect, two pilot studies with a small sample size (11 patients in the study of Zibetti et al. [13] and 7 in our study) point toward similar findings of amelioration of sleep parameters in advanced PD patients treated with LCIG infusion. In our study, however, differences of objective and subjective variables were not statistically significant. The most likely explanation is that the number of subjects ( 7 patients) is insufficient to reach statistical significance in any of the PSG measures presented.

Various studies have demonstrated an improvement in nonmotor symptoms, including the sleep/fatigue domain of the Non-Motor Symptoms Scale (NMSS) and quality of life in advanced PD patients treated with LCIG infusion [11, 25-28]. However, based on a recent systematic review of randomized controlled trials (RCTs) and observational studies collected from PubMed and EMBASE until March 2016, the quality of evidence regarding effectiveness of LCIG infusion in improving quality of life is moderate and for reducing nonmotor symptoms is low [29]. Also, there is no evidence of the effectiveness of LCIG infusion in the treatment of subjective fatigue [30].

There is little information on the specific effect of LCIG infusion on the quality of sleep in patients with advanced PD. In a study by Zibetti et al. [12] carried out in a sample of 12 patients, sleep and nocturnal symptoms were evaluated with the modified version of Parkinson's Disease Sleep Scale (PDSS-2) Daytime sleepiness was also assessed with the ESS. A significant improvement in all study variables (PDSS-2 total score, disturbed sleep, motor symptoms at night, PD symptoms at night, and ESS score) was found at 2-4 months of follow-up after starting LCIG infusion therapy. Honig 
et al. [11] evaluated 24 patients with advanced PD who switched from oral medications to LCIG and were followed for 6 months. Treatment with LCIG reduced motor fluctuations and dyskinesias with statistically significant decreases of the nonmotor symptoms scale (NMSS) and improvement in quality of life (PDQ-8 questionnaire). These results are consistent with the present findings. Although our data should be interpreted considering the reduced number of patients included in the study, it was shown that treatment with LCIG infusion was not associated with a significant amelioration of sleep quality. Overall, the quality of sleep in our patients was poor, but it was not found to be worsened by LCIG infusion therapy.

\section{Conclusions}

The main finding of this preliminary study of 7 advanced PD patients treated with LCGI is a trend toward an improvement of sleep quality after 6 months of LCIG infusion, although differences as compared to pretreatment values were not statistically significant. The sleep architecture was not modified by LCIG. Further prospective masked studies with larger series of patients on LCIG infusion therapy, not stopped at night, are necessary to clarify the positive influence of LCIG on sleep quality in patients with advanced PD.

\section{Data Availability}

The data used to support the findings of this study are available from the corresponding author upon request.

\section{Additional Points}

Additional supporting information may be obtained under request to the correspondence author, Oriol De Fabregues.

\section{Conflicts of Interest}

The authors declare that there are no conflicts of interest.

\section{Authors' Contributions}

ODF designed the study and performed organization and execution; data acquisition; analysis and interpretation of data; manuscript drafting; and manuscript revision. AF and OR performed execution and data acquisition. MQ performed analysis and interpretation of data. JAS revised the manuscript.

\section{Acknowledgments}

The authors thank Marta Pulido, MD, PhD, for editing the manuscript and editorial assistance.

\section{References}

[1] M. Menza, R. D. Dobkin, H. Marin, and K. Bienfait, "Sleep disturbances in Parkinson's disease," Movement Disorders, vol. 25, no. 1, pp. S117-S122, 2010.
[2] A. Iranzo, "Sleep in neurodegenerative diseases," Sleep Medicine Clinics, vol. 11, no. 1, pp. 1-18, 2016.

[3] C. L. Comella, "Sleep disturbances and excessive daytime sleepiness in Parkinson's disease: an overview," Journal of Neural Transmission. Supplementary, vol. 70, pp. 349-355, 2006.

[4] M. R. Najafi, A. Chitsaz, Z. Askarian, and M. A. Najafi, "Quality of sleep in patients with Parkinson's disease," International Journal of Preventive Medicine, vol. 4, no. 2, pp. 229-233, 2013.

[5] T. Scaravilli, E. Gasparoli, F. Rinaldi, G. Polesello, and F. Bracco, "Health-related quality of life and sleep disorders in Parkinson's disease," Neurological Sciences, vol. 24, no. 3, pp. 209-210, 2003.

[6] K. H. Karlsen, E. Tandberg, D. Arsland, and J. P. Larsen, "Health related quality of life in Parkinson's disease: a prospective longitudinal study," Journal of Neurology, Neurosurgery and Psychiatry, vol. 69, no. 5, pp. 584-589, 2000.

[7] P. K. Pal, K. Thennarasu, J. Fleming, M. Schulzer, T. Brown, and S. M. Calne, "Nocturnal sleep disturbances and daytime dysfunction in patients with Parkinson's disease and in their caregivers," Parkinsonism and Related Disorders, vol. 10, no. 3, pp. 157-168, 2004.

[8] A. W. Amara, R. L. Watts, and H. C. Walker, "The effects of deep brain stimulation on sleep in Parkinson's disease," Therapeutic Advances in Neurological Disorders, vol. 4, no. 1, pp. 15-24, 2011.

[9] L. Eugster, P. Bargiotas, C. L. Bassetti, and W. M. M. Schuepbach, "Deep brain stimulation and sleepwake functions in Parkinson's disease: a systematic review," Parkinsonism and Related Disorders, vol. 32, pp. 12-19, 2016.

[10] C. M. Tolleson, K. Bagai, A. S. Walters, and T. L. Davis, "A pilot study assessing the effects of pallidal deep brain stimulation on sleep quality and polysomnography in Parkinson's patients," Neuromodulation: Technology at the Neural Interface, vol. 19, no. 7, pp. 724-730, 2016.

[11] H. Honig, A. Antonini, P. Martinez-Martin et al., "Intrajejunal levodopa infusion in Parkinson's disease: a pilot multicenter study of effects of nonmotor symptoms and quality of life," Movement Disorders, vol. 24, no. 10, pp. 1468-1474, 2009.

[12] M. Zibetti, M. Rizzone, A. Merola et al., "Sleep improvement with levodopa/carbidopa intestinal gel infusion," Acta Neurol Scand, vol. 127, no. 5, pp. e28-e32, 2013.

[13] M. Zibetti, A. Romagnolo, A. Merola et al., "A polysomnographic study in parkinsonian patients treated with intestinal levodopa infusion," Journal of Neurology, vol. 264, no. 6, pp. 1085-1090, 2017.

[14] O. De Fabregues, J. Dot, M. Abu-Suboh et al., "Long-term safety and effectiveness of levodopa-carbidopa intestinal gel infusion," Brain and Behavior, vol. 7, no. 8, article e00758, 2017.

[15] E. Chiner, J. M. Arriero, J. Signes-Costa, J. Marco, and I. Fuentes, "Validación de la versión española del test de somnolencia epworth en pacientes con síndrome de apnea del sueño," Archivos de Bronconeumología, vol. 35, no. 9, pp. 422-427, 1999.

[16] P. O. Valko, C. L. Bassetti, K. E. Bloch, U. Held, and C. R. Baumann, "Validation of the fatigue severity scale in a swiss cohort," Sleep, vol. 31, no. 11, pp. 1601-1607, 2008.

[17] F. Hita-Contreras, E. Martínez-López, P. A. Latorre-Román, F. Garrido, M. A. Santos, and A. Martínez-Amat, "Reliability and validity of the Spanish version of the Pittsburgh sleep quality index (PSQI) in patients with fibromyalgia," Rheumatology International, vol. 34, no. 7, pp. 929-936, 2014. 
[18] J. S. Wiebe and J. A. Penley, "A psychometric comparison of the Beck depression inventory-II in English and Spanish," Psychological Assessment, vol. 17, no. 4, pp. 481-485, 2005.

[19] J. C. Ramon-Brieve, "Validación de la versión castellana de Hamilton para la depresión," Actas Luso Esp Neurol Psiquiatr Cienc Afines, vol. 14, pp. 324-334, 1989.

[20] R. B. Berry, R. Budhiraja, D. J. Gottlieb et al., "Rules for scoring respiratory events in sleep: update of the 2007 AASM manual for the scoring of sleep and associated events. Deliberations of the sleep apnea definitions task force of the American academy of sleep medicine," Journal of Clinical Sleep Medicine, vol. 8, no. 5, pp. 597-619, 2012.

[21] S. Fahn, R. L. Elton, and Members of the UPDRS Development Committee, "Unified Parkinson's disease rating scale," in Recent Developments in Parkinson's Disease, S. Fahn, C. D. Marsden, M. Goldstein et al., Eds., pp. 153-163, Macmillan Healthcare Information, Florham Park, NJ, USA, 1987.

[22] M. M. Hoehn and M. D. Yahr, "Parkinsonism: onset, progression and mortality," Neurology, vol. 17, no. 5, pp. 427-442, 1967.

[23] R. S. Schwab and A. C. England Jr., "Projection technique for evaluating surgery in Parkinson's disease," in Third Symposium on Parkinson's Disease, F. J. Gilingham and I. M. L. Donaldson, Eds., pp. 152-157, E \& S Livingstone, Edinburgh, UK, 1969.

[24] M. F. Folstein, S. E. Folstein, and P. R. McHugh, “"Minimental state": a practical method for grading the cognitive state of patients for the clinician," Journal of Psychiatric Research, vol. 12, no. 3, pp. 189-198, 1975.

[25] M. T. Cáceres-Redondo, F. Carrillo, M. J. Lama et al., "Longterm levodopa/carbidopa intestinal gel in advanced Parkinson's disease," Journal of Neurology, vol. 261, no. 3, pp. 561-569, 2014.

[26] O. Băjenaru, A. Ene, B. O. Popescu et al., "The effect of levodopa-carbidopa intestinal gel infusion long-term therapy on motor complications in advanced Parkinson's disease: a multicenter Romanian experience," Journal of Neural Transmission, vol. 123, no. 4, pp. 407-414, 2016.

[27] A. Antonini, A. Yegin, C. Preda, L. Bergmann, and W. Poewe, "GLORIA study investigators and coordinators. Global longterm study on motor and non-motor symptoms and safety of levodopa-carbidopa intestinal gel in routine care of advanced Parkinson's disease patients; 12-month interim outcomes," Parkinsonism and Related Disorders, vol. 21, no. 3, pp. 231235, 2015.

[28] S. E. Palhagen, N. Dizdar, T. Hauge et al., "Interim analysis of long-term intraduodenal levodopa infusion in advanced Parkinson disease," Acta Neurologica Scandinavica, vol. 126, no. 6, pp. 29-33, 2012.

[29] K. Wirdefeldt, P. Odin, and D. Nyholm, "Levodopa-carbidopa intestinal gel in patients with Parkinson's disease: a systematic review," CNS Drugs, vol. 30, no. 5, pp. 381-404, 2016.

[30] R. G. Elbers, J. Verhoef, E. E. van Wegen, H. W. Berendse, and G. Kwakkel, "Interventions for fatigue in Parkinson's disease," Cochrane Database of Systematic Reviews, vol. 10, article CD010925, 2015. 


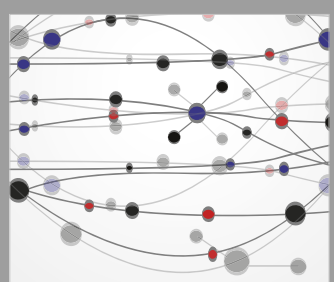

The Scientific World Journal
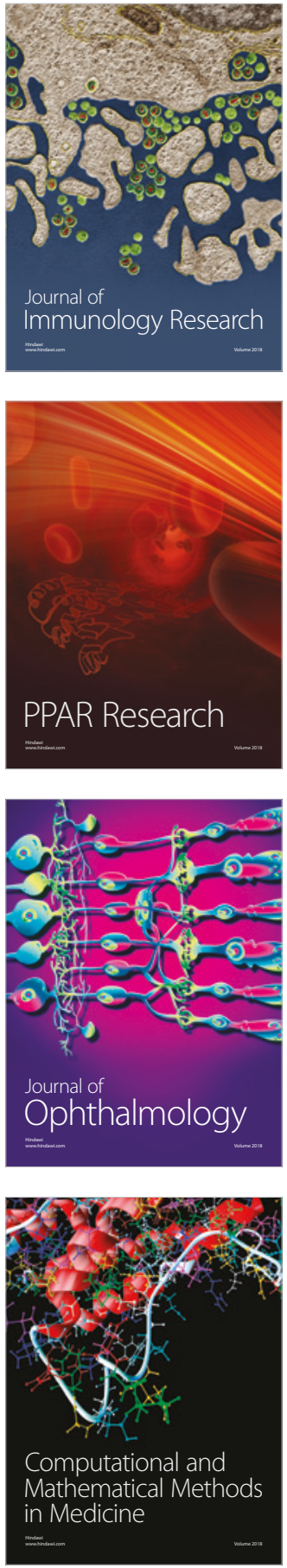

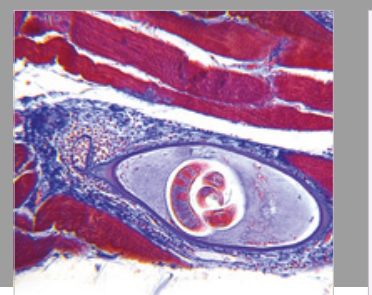

Gastroenterology Research and Practice

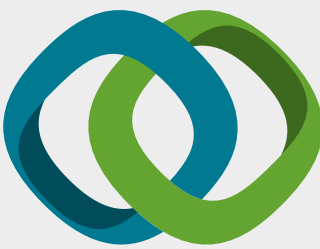

\section{Hindawi}

Submit your manuscripts at

www.hindawi.com
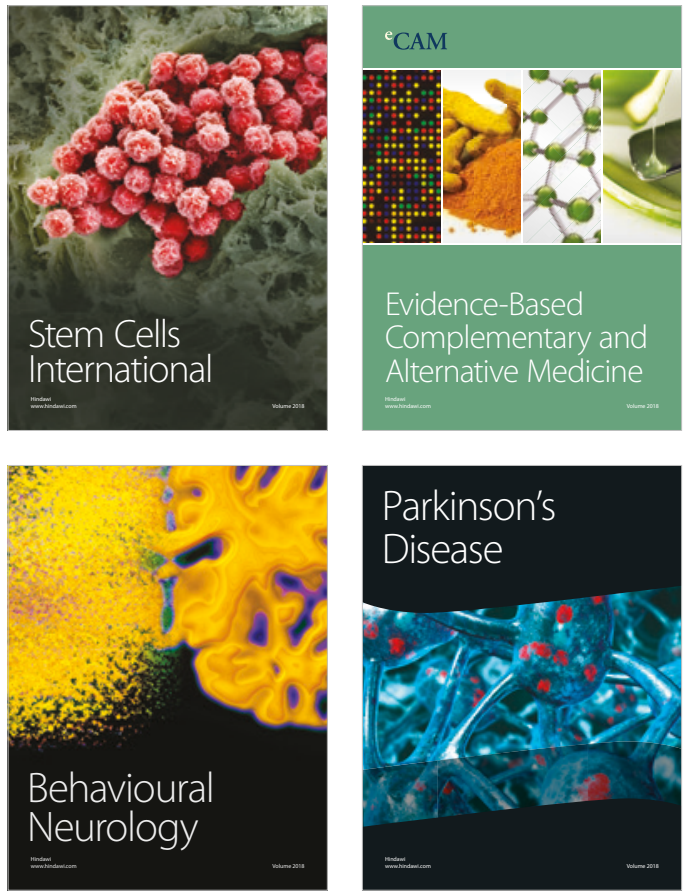

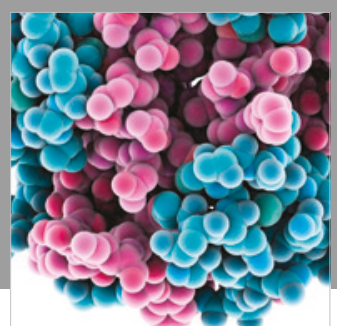

ournal of

Diabetes Research

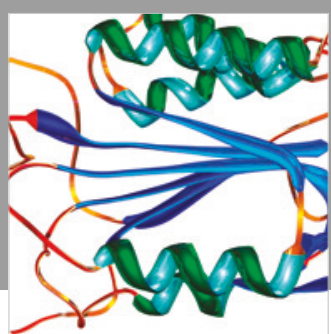

Disease Markers
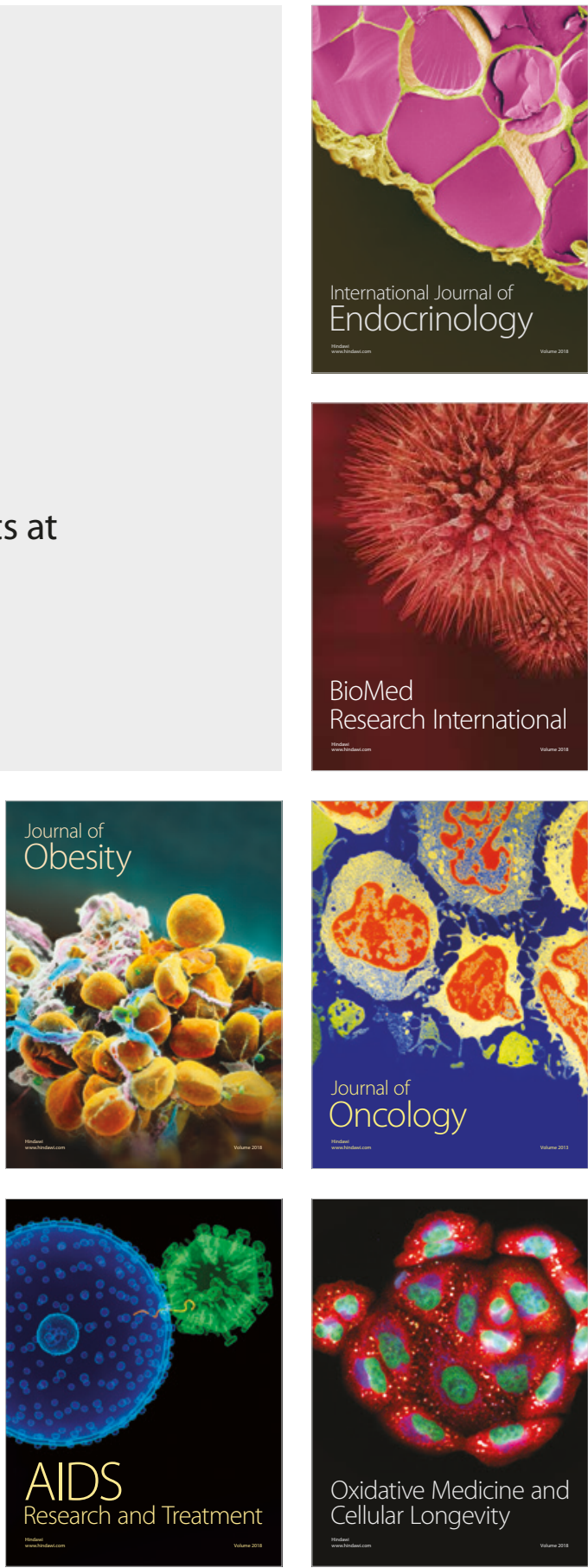